\title{
Circulating lipids and breast cancer prognosis in the Malmö diet and cancer study
}

\author{
Sixten Harborg 1,2 - Thomas P. Ahern ${ }^{3} \cdot$ Maria Feldt $^{4} \cdot$ Ann H. Rosendahl ${ }^{4} \cdot$ Deirdre Cronin-Fenton $^{2}$. \\ Olle Melander ${ }^{5,6,7,8,9} \cdot$ Signe Borgquist ${ }^{1,4}$
}

Received: 18 August 2021 / Accepted: 17 November 2021 / Published online: 25 November 2021

(c) The Author(s) 2021

\begin{abstract}
Purpose Examine the association between circulating lipids and breast cancer outcomes in patients enrolled in the Malmö Diet and Cancer Study (MDCS).

Patients and methods Circulating lipid levels were measured in blood sampled upon enrollment in the female MDCS cohort $(N=17,035)$. We identified all MDCS participants with incident invasive breast cancer diagnosed between 1991 and 2014. Follow-up time began at breast cancer diagnosis and continued until the first event of breast cancer recurrence, death, emigration, or 5 years of follow-up. We estimated the incidence rates of recurrence at 5 years and fit Cox regression models to compute crude and adjusted hazard ratios (HRs) with $95 \%$ confidence intervals $(95 \% \mathrm{CI})$ of breast cancer recurrence as well as all-cause mortality according to cohort-specific tertiles of apolipoprotein A-1 (Apo A-1) and apolipoprotein B (Apo B). Results We enrolled 850 eligible patients. During the 5 years of follow-up, 90 invasive breast cancer recurrences were diagnosed over 3807 person-years. In multivariable analyses, high baseline levels of Apo B were associated with an increased rate of recurrence (tertile 3 vs. $1, \mathrm{HR}=2.30$ [95\% CI 1.13-4.68]). However, high baseline levels of Apo B were not associated with all-cause mortality (tertile 3 vs. $1, \mathrm{HR}=1.23$ [95\% CI 0.68-2.25]). We observed no associations between levels of Apo A-1 and recurrence (tertile 3 vs. $1, \mathrm{HR}=1.34$ [95\% CI 0.70-2.58]) or all-cause mortality (tertile 3 vs. 1 , $\mathrm{HR}=1.12$ [95\% CI 0.61-2.05]).
\end{abstract}

Conclusion High pre-diagnostic levels of Apo B were associated with an increased risk of recurrence among breast cancer patients. Circulating Apo A-1 was not associated with breast cancer outcomes.

Keywords Cancer · Breast cancer · Circulating lipids · Dyslipidemia $\cdot$ Lipids · Apolipoproteins · Apolipoprotein A1 · Apolipoprotein B $\cdot$ Prognosis $\cdot$ Recurrence $\cdot$ Loco-regional recurrence $\cdot$ Distant recurrence $\cdot$ Survival $\cdot$ All-cause mortality $\cdot$ Cohort study

\section{Abbreviations}

Apo A-1 Apolipoprotein A-1

Apo B Apolipoprotein B

BCR Breast cancer recurrence

Sixten Harborg

sixten.harborg@oncology.au.dk

1 Department of Oncology, Aarhus University Hospital/ Aarhus University, Entrance C, Level 1, C118, Palle Juul-Jensens Boulevard 99, 8200 Aarhus N, Denmark

2 Department of Clinical Medicine and Department of Clinical Epidemiology, Aarhus University, Aarhus, Denmark

3 Department of Surgery, Larner College of Medicine, University of Vermont, Burlington, USA

4 Department of Clinical Sciences Lund, Oncology, Skåne University Hospital, Lund University, Lund, Sweden
BCRM Breast cancer-related mortality

LDL Low-density lipoprotein

HDL High-density lipoprotein

27-HC 27-Hydroxycholesterol
5 Department of Clinical Sciences Malmö, Hypertension and Cardiovascular Disease, Lund University, Malmö, Sweden

6 Department of Clinical Sciences Malmö, Lund University Diabetes Centre, Lund University Malmö, Malmö, Sweden

7 Clinical Research Centre, Skåne University Hospital, Lund and Malmö, Malmö, Sweden

8 Department of Emergency and Internal Medicine, Skåne University Hospital, Malmö, Sweden

9 Department of Cardiology, Skåne University Hospital, Malmö, Sweden 


$\begin{array}{ll}\text { MDCS } & \text { Malmö diet and cancer study } \\ \text { ATC } & \text { Anatomical therapeutic chemical codes } \\ \text { BMI } & \text { Body mass index } \\ \text { T1 } & \text { Tertile 1 } \\ \text { T2 } & \text { Tertile 2 } \\ \text { T3 } & \text { Tertile } 3 \\ \text { HR } & \text { Hazard ratio } \\ \text { CI } & \text { Confidence interval }\end{array}$

\section{Introduction}

About 2.1 million women are diagnosed with breast cancer worldwide every year [1]. The survival rates for breast cancer have improved over the last 30 years [2]. However, around $30 \%$ of the women who are declared breast cancer free after primary therapy will eventually present with a recurrence [3]. To improve breast cancer outcomes, there is a need for identifying modifiable causes of breast cancer recurrence $(\mathrm{BCR})$ and breast cancer-related mortality (BCRM) [4].

A meta-analysis of 82 studies concluded that a higher degree of adiposity - a potential modifiable risk factor-is associated with increased risk of BCR and BCRM [5]. Levels of circulating lipids are altered in obese subjects relative to lean controls, and the association between obesity and risk of BCR and BCRM may be partially explained by alterations in circulating lipid levels [6]. An earlier study reported an association between dyslipidemia (defined as abnormal serum levels of total cholesterol and triglycerides) and increased risk of BCRM [7, 8]. Breast cancer cell lines demonstrate alterations in their lipid metabolism compared with normal breast epithelial cells $[9,10]$. This may follow from a greater need for structural lipids in the synthesis of membranes, lipid signaling, and activation of inflammation-related pathways to support tumor survival [11]. The changes in lipid metabolism in cancer are all related to cell growth, proliferation, differentiation, and motility [11].

Cholesterol is an essential lipid for the synthesis of cell membranes [12]. High plasma cholesterol levels have a well-established role in cardiovascular diseases and are associated with an increased risk of and poor prognosis in breast cancer [13]. Cholesterol is transported in the blood via low-density lipoprotein (LDL) and high-density lipoprotein (HDL) [14]; levels of both LDL and HDL have been associated with worse prognosis in breast cancer patients $[15,16]$. However, the results are conflicting [15-18] and need to be further explored. Due to differences in their surface, these lipoproteins contain different classes of apolipoproteins. Apolipoprotein A-1 (Apo A-1) is the most abundant apolipoprotein in HDL [19]. Apo A-1 plays a role in the release of cholesterol from cells and has anti-inflammatory, anti-oxidant, and anti-apoptotic properties [20]. Studies have found that the cholesterol metabolite 27-hydroxycholesterol (27$\mathrm{HC}$ ) is increased when Apo A-1 is overexpressed in mouse models of metastatic breast cancer [21]. Of note, 27-HC is associated with an increase in breast tumor growth and metastasis in estrogen-receptor-positive breast cancer [22]. The role of Apo A-1 in breast cancer has been debated, and the results are conflicting [23-26]. Apolipoprotein B (Apo $\mathrm{B})$ is the major component of LDL [27] and has been wellstudied with regard to cardiovascular diseases [28]. In cancer, experimental studies have linked low tumor expression of Apo B to an increase in metastatic and oncogenic regulators and inhibition of tumor suppressors [29]. Furthermore, high levels of circulating Apo B are associated with diabetes [30], atherogenic disorders [31], and metabolic syndrome [32]; these are all diseases that can affect cancer progression [33]. The association between circulating Apo B levels and breast cancer initiation has been investigated with conflicting results $[25,34]$, but no studies have investigated the link between circulating levels of Apo B and cancer progression.

This study investigated the prognostic role of pre-diagnostic circulating Apo A-1 and Apo B in breast cancer and provides further insight into the interplay between circulating lipids and breast cancer progression. We hypothesized that, compared with low levels of circulating Apo A-1 and Apo B, respectively, high levels of circulating Apo A-1 would be associated with better prognosis of breast cancer, and that high levels of circulating Apo B would be associated with worse prognosis.

\section{Patients and methods}

We conducted a prospective cohort study nested in the Malmö Diet and Cancer Study (MDCS) cohort [35].

\section{Data sources}

The MDCS is a prospective cohort study that enrolled 17,035 women in Malmö, Sweden from 1991 to 1996 [35]. The primary objective of the MDCS was to investigate associations between dietary patterns and cancer risk. The women studied were born between 1923 and 1950 and the MDCS successfully enrolled $42.6 \%$ of the eligible geographic population [36]. Women were not included if they had limited Swedish language skills or disabilities prohibiting completion of study questionnaires. The participants answered extensive questionnaires at baseline and blood samples were collected. Trained research nurses obtained anthropometric measurements including height, weight, and waist/hip circumferences. The MDCS is updated annually with information on incident cancer cases and vital status through record linkage to the Swedish Cancer Registry, the Southern Swedish Regional 
Tumor Registry, and the Swedish Cause of Death Registry [36]. In the National Population Register, a unique civil registration number is assigned to all Swedish residents, which allows the data sources to be linked with $100 \%$ accuracy.

Drug data were retrieved from the Swedish Prescription Registry [37], which collected information on filled prescriptions including anatomical therapeutic chemical codes (ATC) and the date of dispensing at hospitals and pharmacies in Sweden since July 2005. Follow-up data were retrieved from medical records, the Swedish Cause of Death Registry [38], and the Swedish Population Register [39].

\section{Study population}

We identified 1240 female MDCS participants with an incident diagnosis of breast cancer between 1991 and 2014 (Fig. 1). We excluded patients with a breast cancer diagnosis before enrollment in MDCS and patients diagnosed with incident breast carcinoma in situ. Patients were also excluded if they had received neoadjuvant treatment or if metastases were present at breast cancer diagnosis. Likewise, premenopausal patients and patients prescribed cholesterol-lowering statins before their breast cancer diagnosis were excluded because the menopausal transition and statin use both alter apolipoprotein levels. Finally, patients had to have circulating blood lipid levels measured to be included in the survival analyses. After exclusion, 850 patients were included in the survival analysis (Fig. 1).

\section{Apolipoprotein profiles}

Blood samples were retrieved from non-fasting patients at enrollment in the MDCS. Serum and plasma were separated within one hour and stored at $-80^{\circ} \mathrm{C}$; serum concentrations of Apo A-1 and Apo B were measured by Quest Diagnostics (San Juan Capistrano, CA) using an immunonephelometric assay run on the Siemens BNII (Siemens, Newark, DE). The technicians were blinded to cancer status. The interassay variability for Apo A-1 and Apo B was below 4\%. Further details about the treatment of blood samples have been described previously [35, 40].

\section{Definitions of analytic variables}

We grouped Apo A-1 into tertiles according to the distribution of blood levels in the study-specific cohort of MDCS: tertile $1(<154 \mathrm{mg} / \mathrm{dl})$, tertile $2(154-177 \mathrm{mg} / \mathrm{dl})$, and tertile $3(>177 \mathrm{mg} / \mathrm{dl})$. These groups were modeled as categorical variables with the lowest tertile as the reference group. The same approach was used to define tertiles of Apo B: tertile $1(<90 \mathrm{mg} / \mathrm{dl})$, tertile $2(90-110 \mathrm{mg} / \mathrm{dl})$, and tertile 3 $(>110 \mathrm{mg} / \mathrm{dl})$. Likewise, Apo B was included in the models as a categorical variable with the lowest tertile as the reference group. Patients in tertile 1 had low levels, patients in tertile 2 had medium levels, and patients in tertile 3 had high levels of either Apo A-1 or Apo B.

Age was described in decade categories at enrollment in MDCS. In the multivariable models, age was included as a continuous variable defined at the time of breast cancer diagnosis. We used the World Health Organization's body mass index (BMI) definitions [41]: normal-weight
Fig. 1 Flowchart of the study cohort

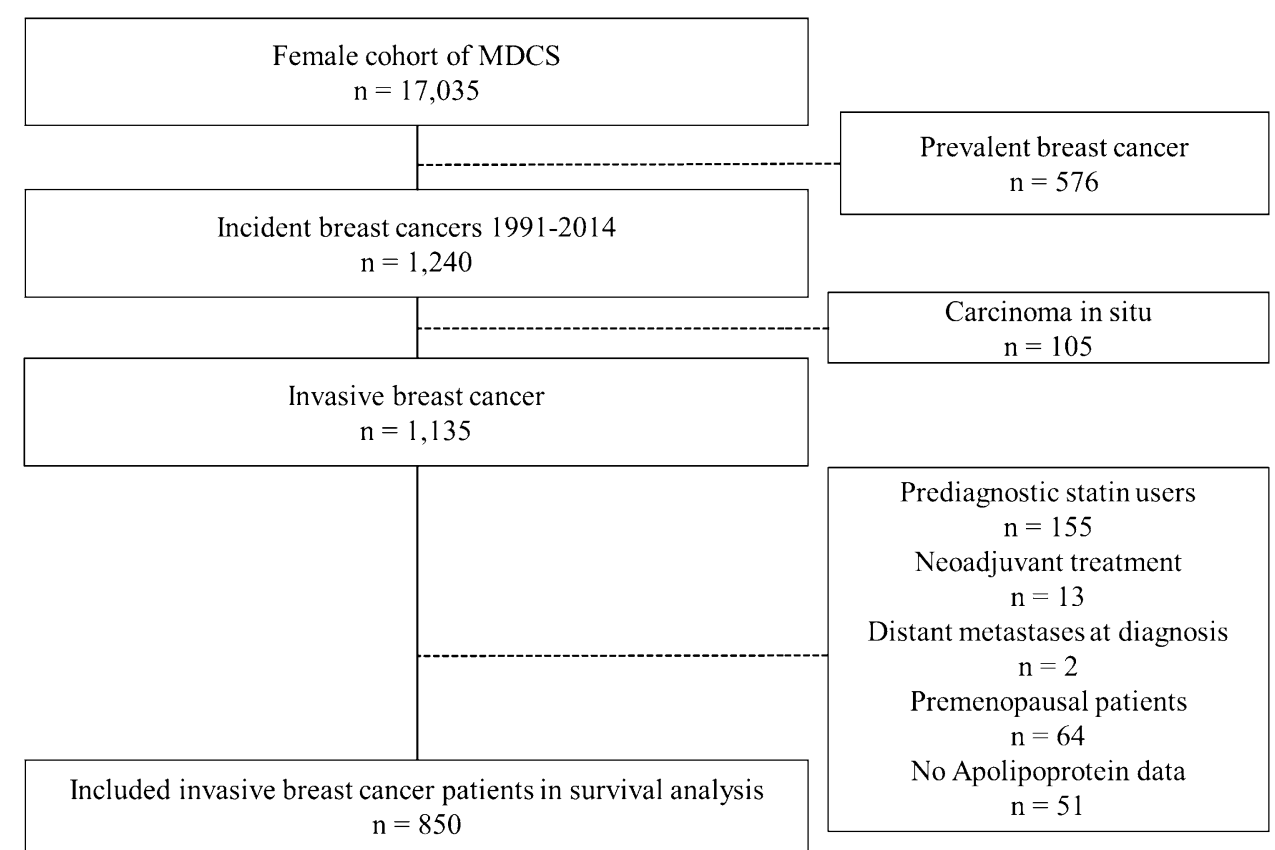


(BMI $<25 \mathrm{~kg} / \mathrm{m}^{2}$ ), overweight $\left(\mathrm{BMI} \geq 25\right.$ and $<30 \mathrm{~kg} / \mathrm{m}^{2}$ ), obese (BMI $\geq 30 \mathrm{~kg} / \mathrm{m}^{2}$ ). BMI was included in the regression models as a continuous variable and finer adjustment with factor variables did not affect the impact of adjustment for BMI on the main exposure term. Patients were further described by three categories of waist circumference in centimeters $(<81,81-85$, and $>85)$ and waist/hip ratio $(\leq 0.80$, $0.81-0.84$, and $\geq 0.85$ ).

We defined three categories of tumor size in millimeters (mm): less than $10 \mathrm{~mm}, 10-20 \mathrm{~mm}$, and more than $20 \mathrm{~mm}$ [42]; tumor size was modeled as a categorical variable. The histological grade of breast cancers was assessed and described according to the Nottingham histological score [43]. Histological grade was categorized as grade I (well differentiated [3-5 points]), grade II (moderately differentiated [6-7 points]), and grade III (poorly differentiated [8-9 points]); this was modeled as a categorical variable. Post-diagnostic statin use was included in the model as a dichotomous time-varying variable, and one filled prescription for statins was assumed to expose the individual from the prescription date through the remaining follow-up time. Estrogen-receptor status (positive/negative), lymph node status (positive [present metastatic lymph nodes]/negative [no metastatic lymph nodes]), surgery (lumpectomy/mastectomy) as well as intended adjuvant treatment with radiotherapy (yes/no), chemotherapy (yes/no), and endocrine therapy (yes/no) were treated as dichotomous variables.

\section{Outcome}

Our primary endpoint was breast cancer recurrence, defined as time from breast cancer diagnosis until the earliest occurrence of invasive loco-regional recurrence, distant metastasis, invasive contralateral breast cancer, or second primary breast cancer. Distant recurrence was defined as time from breast cancer diagnosis until occurrence of metastasis, invasive contralateral breast cancer, or second primary breast cancer. Loco-regional recurrence was defined as time from breast cancer diagnosis until occurrence of recurrence without signs of metastatic disease. Trained personnel retrieved data on recurrence from medical charts of all patients diagnosed with breast cancer. Our secondary endpoint was allcause mortality. Information regarding cause of death was retrieved using the Swedish Cause of Death Registry and defined as time from breast cancer diagnosis until death.

\section{Follow-up and statistical analysis}

Follow-up for breast cancer recurrence and all-cause mortality began at the time of breast cancer diagnosis and continued until the first event of breast cancer recurrence, death from any cause, emigration, or 5 years of follow-up. Patients with these events were censored at the time of event.
We estimated incidence rates of BCR at 5 years and used Cox regression models to compute crude and adjusted hazard ratios (HRs) with $95 \%$ confidence intervals $(95 \% \mathrm{CI}$ ) for both breast cancer recurrence (BCR) and all-cause mortality. Only patients with complete data on all regressed variables were included in the analyses. The adjusted model included the following covariables: age at diagnosis (continuous), body mass index (continuous), node status (dichotomous), estrogen-receptor status (dichotomous), histological grade (categorical), tumor size (categorical), adjuvant radiotherapy (dichotomous), adjuvant chemotherapy (dichotomous), adjuvant endocrine therapy (dichotomous), type of surgery (dichotomous), and post-diagnostic use of statins (dichotomous, time-varying). In the crude analyses, 850 patients were included. Complete case data were available for 650 patients, which were included in the adjusted models. We conducted a series of sensitivity analyses. First, we examined possible differences in types of BCR using Cox regression models to compute HRs and 95\% CI of loco-regional recurrence and distant recurrence. Second, as a large proportion of breast cancers recur late [44], we performed analyses where follow-up beyond 5 years was allowed. Finally, to examine possible differences in the association between apolipoproteins and breast cancer prognosis according to patterns of breast cancer detection, we conducted analyses stratified for the detection of breast cancer through screening mammography or clinical detection.

\section{Results}

In the cohort of 850 patients with incident postmenopausal breast cancer, the median age at diagnosis was 56.3 years (interquartile range was 50.3-62.4). Most breast cancers in this study were detected through mammography screening (screening-detected, $n=436$ vs clinically detected, $n=406$ ). The median recurrence follow-up was 5 years and the total person-years of recurrence follow-up was 3807, during which 90 recurrences occurred: 11 loco-regional and 79 distant.

Patients with high levels of Apo A-1 appeared more likely to have axillary lymph node involvement at diagnosis versus patients with low Apo A-1 levels. Further, patients with high levels of Apo A-1 were more frequently normal-weight, had a smaller waist circumference, and a lower waist/hip ratio. Most patients underwent lumpectomy, however, patients with high levels of Apo A-1 were more frequently appointed mastectomy. Patients with high levels of Apo A-1 were also less frequently prescribed statins (Table 1).

Patients with high levels of Apo B had higher histological grade and larger tumors. In contrast to patients with high levels of Apo A-1, patients with high levels of Apo B were older and more often overweight or obese. Patients with high 
Table 1 Patient characteristics according to Apolipoprotein A-1 tertiles

\begin{tabular}{|c|c|c|c|c|}
\hline & $\begin{array}{l}\text { Total } \\
N=850\end{array}$ & $\begin{array}{l}1(<154 \mathrm{mg} / \mathrm{dl}) \\
N=292\end{array}$ & $\begin{array}{l}2(154-177 \mathrm{mg} / \mathrm{dl}) \\
N=286\end{array}$ & $\begin{array}{l}3(>177 \mathrm{mg} / \mathrm{dl}) \\
N=272\end{array}$ \\
\hline \multicolumn{5}{|l|}{ Age (baseline) } \\
\hline$<50$ & $193(22.7 \%)$ & $78(26.7 \%)$ & $59(20.6 \%)$ & $56(20.6 \%)$ \\
\hline $50-59$ & $359(42.2 \%)$ & $118(40.4 \%)$ & $122(42.7 \%)$ & $119(43.8 \%)$ \\
\hline $60-69$ & $243(28.6 \%)$ & $74(25.3 \%)$ & $81(28.3 \%)$ & $88(32.4 \%)$ \\
\hline$\geq 70$ & $55(6.5 \%)$ & $22(7.5 \%)$ & $24(8.4 \%)$ & $9(3.3 \%)$ \\
\hline \multicolumn{5}{|l|}{ Histological grade } \\
\hline Grade I & $222(28.5 \%)$ & $82(30.6 \%)$ & $67(25.3 \%)$ & $73(29.6 \%)$ \\
\hline Grade II & $363(46.5 \%)$ & $116(43.3 \%)$ & $132(49.8 \%)$ & $115(46.6 \%)$ \\
\hline Grade III & $195(25.0 \%)$ & $70(26.1 \%)$ & $66(24.9 \%)$ & $59(23.9 \%)$ \\
\hline \multicolumn{5}{|l|}{ Tumor size (mm) } \\
\hline$<10$ & $151(18.6 \%)$ & $53(19.1 \%)$ & $47(17.2 \%)$ & $51(19.5 \%)$ \\
\hline 10-20 & $435(53.6 \%)$ & $140(50.5 \%)$ & $151(55.3 \%)$ & $144(55.0 \%)$ \\
\hline$>20$ & $226(27.8 \%)$ & $84(30.3 \%)$ & $75(27.5 \%)$ & $67(25.6 \%)$ \\
\hline \multicolumn{5}{|l|}{ Node status } \\
\hline Negative & $526(69.3 \%)$ & $175(68.9 \%)$ & $190(72.8 \%)$ & $161(66.0 \%)$ \\
\hline Positive & $233(30.7 \%)$ & $79(31.1 \%)$ & $71(27.2 \%)$ & $83(34.0 \%)$ \\
\hline \multicolumn{5}{|c|}{ Estrogen-receptor status } \\
\hline Negative & $93(12.3 \%)$ & $24(9.3 \%)$ & $37(14.2 \%)$ & $32(13.4 \%)$ \\
\hline Positive & $663(87.7 \%)$ & $233(90.7 \%)$ & $224(85.8 \%)$ & $206(86.6 \%)$ \\
\hline \multicolumn{5}{|l|}{ Body mass index } \\
\hline Normal-weight & $436(51.3 \%)$ & $122(41.8 \%)$ & $144(50.3 \%)$ & $170(62.5 \%)$ \\
\hline Overweight & $298(35.1 \%)$ & $119(40.8 \%)$ & $96(33.6 \%)$ & $83(30.5 \%)$ \\
\hline Obese & $116(13.6 \%)$ & $51(17.5 \%)$ & $46(16.1 \%)$ & $19(7.0 \%)$ \\
\hline \multicolumn{5}{|l|}{ Waist/hip ratio } \\
\hline$\leq 0.80$ & $531(62.5 \%)$ & $159(54.6 \%)$ & $175(61.2 \%)$ & $197(72.4 \%)$ \\
\hline $0.81-0.84$ & $205(24.1 \%)$ & $83(28.5 \%)$ & $67(23.4 \%)$ & $55(20.2 \%)$ \\
\hline$\geq 0.85$ & $113(13.3 \%)$ & $49(16.8 \%)$ & $44(15.4 \%)$ & $20(7.4 \%)$ \\
\hline \multicolumn{5}{|c|}{ Waist circumference $(\mathrm{cm})$} \\
\hline$<81$ & $563(66.3 \%)$ & $166(57.0 \%)$ & $187(65.4 \%)$ & $210(77.2 \%)$ \\
\hline $81-85$ & $109(12.8 \%)$ & $52(17.9 \%)$ & $26(9.1 \%)$ & $31(11.4 \%)$ \\
\hline$>85$ & $177(20.8 \%)$ & $73(25.1 \%)$ & $73(25.5 \%)$ & $31(11.4 \%)$ \\
\hline \multicolumn{5}{|c|}{ Surgical procedure } \\
\hline Lumpectomy & $465(59.3 \%)$ & $163(59.5 \%)$ & $162(63.3 \%)$ & $140(55.1 \%)$ \\
\hline Mastectomy & $319(40.7 \%)$ & $111(40.5 \%)$ & $94(36.7 \%)$ & $114(44.9 \%)$ \\
\hline \multicolumn{5}{|c|}{ Endocrine therapy, planned adjuvant } \\
\hline No & $363(44.3 \%)$ & $122(43.9 \%)$ & $123(44.7 \%)$ & $118(44.4 \%)$ \\
\hline Yes & $456(55.7 \%)$ & $156(56.1 \%)$ & $152(55.3 \%)$ & $148(55.6 \%)$ \\
\hline \multicolumn{5}{|c|}{ Chemotherapy, planned adjuvant } \\
\hline No & $659(85.5 \%)$ & $222(85.1 \%)$ & $231(88.8 \%)$ & $206(82.4 \%)$ \\
\hline Yes & $112(14.5 \%)$ & $39(14.9 \%)$ & $29(11.2 \%)$ & $44(17.6 \%)$ \\
\hline \multicolumn{5}{|c|}{ Radiotherapy, planned adjuvant } \\
\hline No & $323(41.7 \%)$ & $109(41.8 \%)$ & $105(40.1 \%)$ & $109(43.3 \%)$ \\
\hline Yes & $452(58.3 \%)$ & $152(58.2 \%)$ & $157(59.9 \%)$ & $143(56.7 \%)$ \\
\hline \multicolumn{5}{|c|}{ Postdiagnostic statin use } \\
\hline No & $751(88.4 \%)$ & $249(85.3 \%)$ & $254(88.8 \%)$ & $248(91.2 \%)$ \\
\hline Yes & $99(11.6 \%)$ & $43(14.7 \%)$ & $32(11.2 \%)$ & $24(8.8 \%)$ \\
\hline
\end{tabular}


Apo B levels were also more often appointed lumpectomy and more frequently prescribed statins than patients with low levels of Apo B (Table 2).

Table 3 presents the estimated recurrence and mortality hazard ratios in relation to Apo A-1 tertiles. There were 29 recurrences in tertile 1 (T1) during 1324 person-years of follow-up, 29 recurrences in tertile 2 (T2) in 1257 personyears, and 32 recurrences occurred in 1224 person-years of follow-up in tertile 3 (T3). No strong associations were seen between levels of Apo A-1 and BCR (T3, adj HR: 1.34 [95\% CI 0.70-2.58]), distant recurrence (T3, adjHR: 1.29 [95\% CI 0.66-2.50]), loco-regional recurrence (T3, ${ }_{\text {adj }} \mathrm{HR}: 1.19$ [95\% CI 0.30-4.75]), or all-cause mortality (T3, adj HR: 1.12 [95\% CI 0.61-2.05]) (Table 3, Fig. 2). Neither were any association between Apo A-1 and BCR observed in analyses stratified for detection mode of breast cancer through mammography screening (T3, adj HR: 1.06 [95\% CI 0.36-3.15]) or clinical detection (T3, adj HR: 1.40 [95\% CI 0.59-3.34]) (Table 5).

Table 4 displays estimated recurrence and mortality hazard ratios in relation to Apo B tertiles. There were 17 recurrences among patients in $\mathrm{T} 1$ during 1304 person-years of follow-up, 32 recurrences in T2 in 1293 person-years, and 41 recurrences in 1209 person-years of follow-up in T3. In univariable analyses, high levels of Apo B were associated with BCR (T3, crude HR: 2.69 [95\% CI 1.51-4.81]) and distant recurrence (T3, crude HR: 2.61 [95\% CI 1.40-4.87]). High levels of Apo B were also positively associated with BCR (T3, adj HR: 2.30 [95\% CI 1.13-4.68]) and distant recurrence (T3, adj HR: 2.19 [95\% CI 1.02-4.70]) in multivariable analyses. However, high levels of Apo B were not associated with an increased risk of loco-regional recurrence (T3, adj HR: 0.73 [95\% CI 0.16-3.27]) or all-cause mortality (T3, adj HR: 1.23 [95\% CI 0.68-2.25]) (Table 4, Fig. 2). In analyses allowing a longer follow-up than 5 years, the positive association between high levels of Apo B and BCR was consistent (T3,

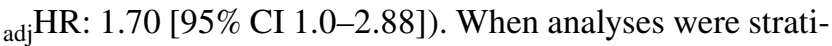
fied for primary breast cancer mode of detection, the association between Apo B and BCR was attenuated for patients detected through mammography screening (T3, adjHR: 1.01 [95\% CI 0.31-3.28]), while the association was persistent for patients with a clinically detected breast cancer $\left(\mathrm{T} 3,{ }_{\text {adj }} \mathrm{HR}\right.$ : 3.87 [95\% CI 1.39-10.75]) (Table 5).

\section{Discussion}

This observational study showed a positive association between high levels of pre-diagnostic Apo B and breast cancer recurrence. This association remained positive for distant recurrences but not for loco-regional recurrences. In the 5-year estimates, patients with high levels of Apo B had a two-fold increased risk of recurrence of their breast cancer versus patients with low levels of Apo B. In contrast, there was no association between Apo A-1 levels and breast cancer outcomes. To the best of our knowledge, this is the first study to evaluate if Apo A-1 and Apo B are associated with recurrence in breast cancer patients.

Our study has some limitations. First, the measurements of circulating apolipoprotein levels were obtained from baseline serum specimens and were drawn well before breast cancer diagnosis. This might induce a misclassification of apolipoprotein levels at etiologically appropriate time periods because apolipoprotein levels can be modulated, e.g., by lifestyle changes, statins, or entering menopause. To minimize the risk of misclassification and time-dependent confounding, we excluded all premenopausal patients and patients who were prescribed statins before their breast cancer diagnosis. However, we cannot exclude the possibility that lipid levels may have changed between blood draw and breast cancer diagnosis; our findings may therefore still be prone to misclassification.

Second, many patients received adjuvant endocrine therapy, and endocrine therapy might modulate circulating apolipoprotein levels differently, e.g., tamoxifen increases Apo A-1 [45] and aromatase inhibitors increase Apo B [46]. Thus, it could have been of value to account for changes in blood levels of apolipoproteins during endocrine therapy. However, this was unfortunately not possible because postdiagnostic blood samples (for Apo A-1 and Apo B analysis) and data on changes in endocrine therapy during followup were not available. In a sensitivity analysis stratified by planned endocrine therapy, the positive association between high levels of Apo B and recurrence was only observed for patients receiving endocrine therapy (T3, HR: 3.30 [95\% CI 1.22-8.92]).

Third, apolipoprotein levels follow systemic cholesterol levels, and high levels of cholesterol derivatives are associated with poor breast cancer prognosis [22]. The association between Apo B and increased risk of BCR might therefore be driven by other harmful prognostic factors correlated with Apo B such as high blood levels of LDL that have previously been reported to be associated with worse disease-free survival in breast cancer [15]. Blood levels of total cholesterol, HDL and LDL were only collected for a small sub-population in MDCS. However, the size of the sub-population limits statistical analyses and assessment of their importance concerning prognosis in this study.

Finally, it should be acknowledged that there might exist positive confounders, which we were not able to adjust for in our statistical models. Hence, our results might be affected by residual confounding as the association between Apo B and breast cancer recurrence was attenuated in multivariable models.

Apo B's relation to 27-HC and LDL might explain the potential mechanism linking Apo B levels to a worse 
Table 2 Patient characteristics according to Apolipoprotein B tertiles

\begin{tabular}{|c|c|c|c|c|}
\hline & $\begin{array}{l}\text { Total } \\
N=850\end{array}$ & $\begin{array}{l}1(<90 \mathrm{mg} / \mathrm{dl}) \\
N=286\end{array}$ & $\begin{array}{l}2(90-110 \mathrm{mg} / \mathrm{dl}) \\
N=284\end{array}$ & $\begin{array}{l}3(>110 \mathrm{mg} / \mathrm{dl}) \\
N=280\end{array}$ \\
\hline \multicolumn{5}{|l|}{ Age (baseline) } \\
\hline$<50$ & $193(22.7 \%)$ & $104(36.4 \%)$ & $62(21.8 \%)$ & $27(9.6 \%)$ \\
\hline $50-59$ & $359(42.2 \%)$ & $121(42.3 \%)$ & $126(44.4 \%)$ & $112(40.0 \%)$ \\
\hline $60-69$ & $243(28.6 \%)$ & $56(19.6 \%)$ & $71(25.0 \%)$ & $116(41.4 \%)$ \\
\hline$\geq 70$ & $55(6.5 \%)$ & $5(1.7 \%)$ & $25(8.8 \%)$ & $25(8.9 \%)$ \\
\hline \multicolumn{5}{|l|}{ Histological grade } \\
\hline Grade I & $222(28.5 \%)$ & $88(34.1 \%)$ & $69(26.2 \%)$ & $65(25.1 \%)$ \\
\hline Grade II & $363(46.5 \%)$ & $112(43.4 \%)$ & $132(50.2 \%)$ & $119(45.9 \%)$ \\
\hline Grade III & $195(25.0 \%)$ & $58(22.5 \%)$ & $62(23.6 \%)$ & $75(29.0 \%)$ \\
\hline \multicolumn{5}{|l|}{ Tumor size (mm) } \\
\hline$<10$ & $151(18.6 \%)$ & $52(19.0 \%)$ & $64(23.4 \%)$ & $35(13.3 \%)$ \\
\hline 10-20 & $435(53.6 \%)$ & $158(57.7 \%)$ & $138(50.4 \%)$ & $139(52.7 \%)$ \\
\hline$>20$ & $226(27.8 \%)$ & $64(23.4 \%)$ & $72(26.3 \%)$ & $90(34.1 \%)$ \\
\hline \multicolumn{5}{|l|}{ Node status } \\
\hline Negative & $526(69.3 \%)$ & $189(73.3 \%)$ & $179(69.1 \%)$ & $158(65.3 \%)$ \\
\hline Positive & $233(30.7 \%)$ & $69(26.7 \%)$ & $80(30.9 \%)$ & $84(34.7 \%)$ \\
\hline \multicolumn{5}{|c|}{ Estrogen-receptor status } \\
\hline Negative & $93(12.3 \%)$ & $26(10.4 \%)$ & $30(11.7 \%)$ & $37(14.9 \%)$ \\
\hline Positive & $663(87.7 \%)$ & $225(89.6 \%)$ & $226(88.3 \%)$ & $212(85.1 \%)$ \\
\hline \multicolumn{5}{|l|}{ Body mass index } \\
\hline Normal-weight & $436(51.3 \%)$ & $185(64.7 \%)$ & $153(53.9 \%)$ & $98(35.0 \%)$ \\
\hline Overweight & $298(35.1 \%)$ & $78(27.3 \%)$ & $104(36.6 \%)$ & $116(41.4 \%)$ \\
\hline Obese & $116(13.6 \%)$ & $23(8.0 \%)$ & $27(9.5 \%)$ & $66(23.6 \%)$ \\
\hline \multicolumn{5}{|l|}{ Waist/hip ratio } \\
\hline$\leq 0.80$ & $531(62.5 \%)$ & $212(74.4 \%)$ & $185(65.1 \%)$ & $134(47.9 \%)$ \\
\hline $0.81-0.84$ & $205(24.1 \%)$ & $50(17.5 \%)$ & $65(22.9 \%)$ & $90(32.1 \%)$ \\
\hline$\geq 0.85$ & $113(13.3 \%)$ & $23(8.1 \%)$ & $34(12.0 \%)$ & $56(20.0 \%)$ \\
\hline \multicolumn{5}{|c|}{ Waist circumference $(\mathrm{cm})$} \\
\hline$<81$ & $563(66.3 \%)$ & $229(80.4 \%)$ & $197(69.4 \%)$ & $137(48.9 \%)$ \\
\hline $81-85$ & $109(12.8 \%)$ & $27(9.5 \%)$ & $41(14.4 \%)$ & $41(14.6 \%)$ \\
\hline$>85$ & $177(20.8 \%)$ & $29(10.2 \%)$ & $46(16.2 \%)$ & $102(36.4 \%)$ \\
\hline \multicolumn{5}{|c|}{ Surgical procedure } \\
\hline Lumpectomy & $465(59.3 \%)$ & $163(61.3 \%)$ & $164(61.7 \%)$ & $138(54.8 \%)$ \\
\hline Mastectomy & $319(40.7 \%)$ & $103(38.7 \%)$ & $102(38.3 \%)$ & $114(45.2 \%)$ \\
\hline \multicolumn{5}{|c|}{ Endocrine therapy, planned adjuvant } \\
\hline No & $363(44.3 \%)$ & $119(43.0 \%)$ & $131(47.8 \%)$ & $113(42.2 \%)$ \\
\hline Yes & $456(55.7 \%)$ & $158(57.0 \%)$ & $143(52.2 \%)$ & $155(57.8 \%)$ \\
\hline \multicolumn{5}{|c|}{ Chemotherapy, planned adjuvant } \\
\hline No & $659(85.5 \%)$ & $218(84.8 \%)$ & $222(85.4 \%)$ & $219(86.2 \%)$ \\
\hline Yes & $112(14.5 \%)$ & $39(15.2 \%)$ & $38(14.6 \%)$ & $35(13.8 \%)$ \\
\hline \multicolumn{5}{|c|}{ Radiotherapy, planned adjuvant } \\
\hline No & $323(41.7 \%)$ & $106(41.2 \%)$ & $99(37.9 \%)$ & $118(45.9 \%)$ \\
\hline Yes & $452(58.3 \%)$ & $151(58.8 \%)$ & $162(62.1 \%)$ & $139(54.1 \%)$ \\
\hline \multicolumn{5}{|c|}{ Postdiagnostic statin use } \\
\hline No & $751(88.4 \%)$ & $271(94.8 \%)$ & $247(87.0 \%)$ & $233(83.2 \%)$ \\
\hline Yes & $99(11.6 \%)$ & $15(5.2 \%)$ & $37(13.0 \%)$ & $47(16.8 \%)$ \\
\hline
\end{tabular}


Table 3 Apolipoprotein A-1 tertiles in relation to recurrence and survival
Fig. 2 Cumulative incidence of recurrence in breast cancer according to apolipoprotein tertiles in patients enrolled in MDCS

\begin{tabular}{ccclll}
\hline Endpoint & Tertiles & Events & Person-years & Unadjusted HR (95\% CI) & Adjusted HR (95\% CI ${ }^{\mathrm{a}}$ \\
\hline Any recurrence & & & & \\
T1 & 29 & 1324.83 & $($ Ref $)$ & \\
T2 & 29 & 1257.82 & $1.05(0.62-1.78)$ & $1.20(0.62-2.32)$ \\
T3 & 32 & 1224.85 & $1.19(0.72-1.99)$ & $1.34(0.70-2.58)$ \\
Distant recurrence & & & & \\
T1 & 25 & 356.49 & $($ Ref $)$ & $1.20(0.62-2.32)$ \\
T2 & 27 & 370.52 & $1.14(0.65-1.97)$ & $1.26(0.65-2.45)$ \\
T3 & 28 & 428.93 & $1.25(0.73-2.16)$ & \\
All-cause mortality & & & & $1.11(0.63-1.98)$ \\
T1 & 35 & 1135.72 & $(\operatorname{Ref})$ & $1.12(0.61-2.05)$ \\
T2 & 45 & 860.90 & $1.34(0.86-2.08)$ & \\
T3 & 39 & 923.52 & $1.18(0.75-1.87)$ & \\
\hline
\end{tabular}

${ }^{a}$ Adjusted for age, body mass index, estrogen-receptor status, histological grade, nodal status, tumor size, adjuvant endocrine treatment, chemotherapy, radiotherapy, surgery and statins

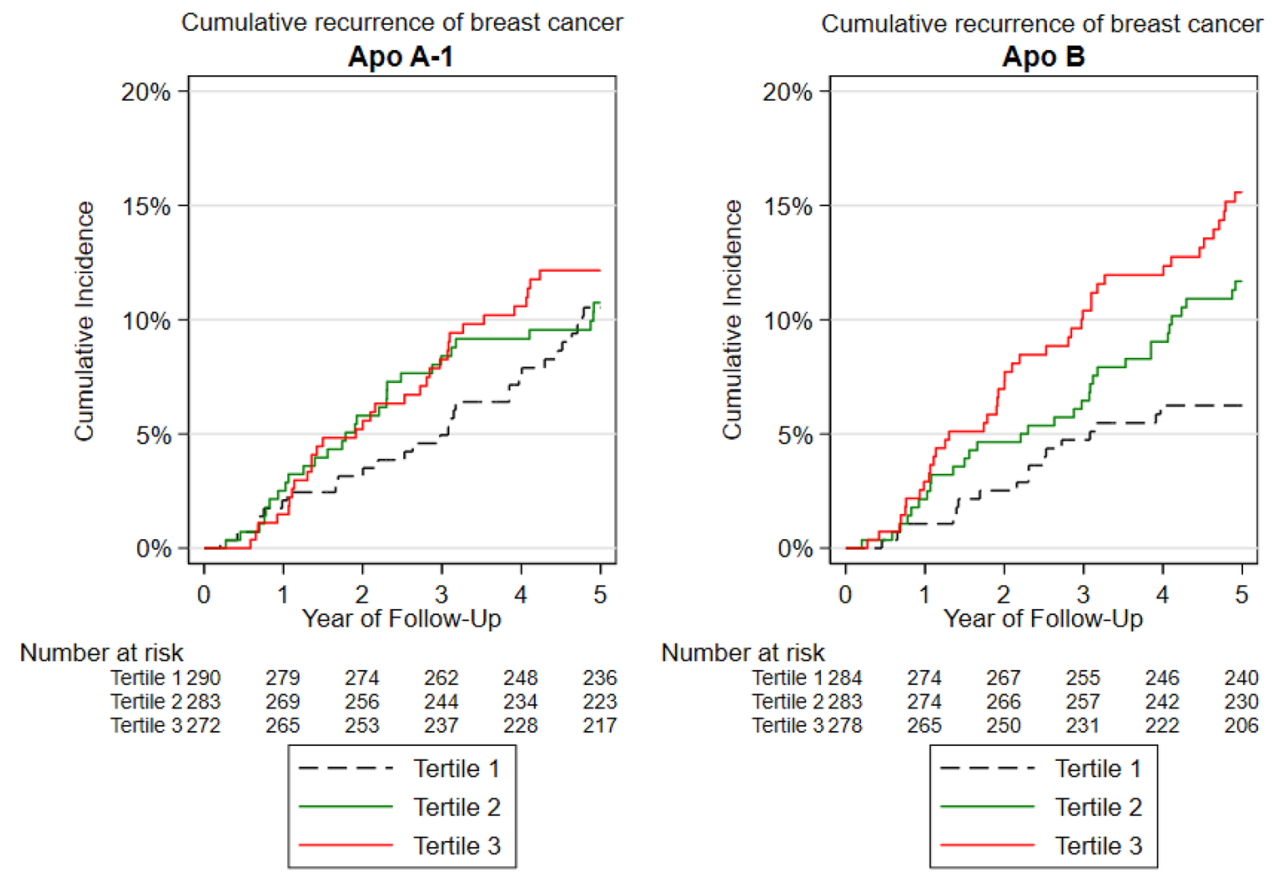

outcome in breast cancer-27-HC is primarily transported by LDL and is associated with an increase in breast tumor growth and metastasis in estrogen-receptor-positive breast cancer [22]. Apo B is the main protein constituent of LDL and Apo B thus mirrors LDL levels in serum. The negative prognostic value of high Apo B levels might therefore be explained by the function of Apo B levels as a surrogate marker of 27-HC levels via LDL. Yet, investigating possible differences in Apo B's association with BCR according to estrogen-receptor status did not explain the association further. Thus, the positive association between Apo B and BCR was not considered limited to a certain subtype of breast cancer in this study.
The difference in association between Apo B levels and outcome observed among patients with primary breast cancer detected through mammography screening and clinical detection may partially be explained by the relationship between Apo B and breast density. Breast density is linked to a higher risk of breast cancer [47], at the same time high breast density makes breast cancers harder to detect through mammography [48]. Previous research has shown an association between low levels of Apo B and high breast density [49]. This would suggest that patients with high levels of Apo B are to be diagnosed with breast cancer earlier than those with low levels as these breast cancers would be harder to identify through mammography screening. Yet, in this 
Table 4 Apolipoprotein B tertiles in relation to recurrence and survival

\begin{tabular}{ccclll}
\hline Endpoint & Tertiles & Events & Person-years & Unadjusted HR (95\% CI) & Adjusted HR (95\% CI ${ }^{\mathrm{a}}$ \\
\hline Any recurrence & & & & \\
T1 & 17 & 1304.19 & $($ Ref $)$ & \\
T2 & 32 & 1293.50 & $1.96(1.07-3.58)$ & $1.70(0.82-3.53)$ \\
T3 & 41 & 1209.82 & $2.69(1.51-4.81)$ & $2.30(1.13-4.68)$ \\
Distant recurrence & & & & \\
T1 & 15 & 348.64 & $($ Ref $)$ & $1.61(0.74-3.52)$ \\
T2 & 30 & 448.68 & $2.15(1.14-4.06)$ & $2.19(1.02-4.70)$ \\
T3 & 35 & 358.62 & $2.61(1.40-4.87)$ & \\
All-cause mortality & & & & $0.81(0.42-1.56)$ \\
T1 & 35 & 670.84 & $($ Ref $)$ & $1.23(0.68-2.25)$ \\
T2 & 34 & 1033.77 & $0.97(0.61-1.56)$ & \\
T3 & 50 & 1215.53 & $1.52(0.98-2.34)$ &
\end{tabular}

${ }^{a}$ Adjusted for age, body mass index, estrogen-receptor status, histological grade, nodal status, tumor size, adjuvant endocrine treatment, chemotherapy, radiotherapy, surgery and statins

Table 5 Primary detection and apolipoproteins in relation to recurrence

\begin{tabular}{|c|c|c|c|c|c|c|c|}
\hline \multirow{2}{*}{$\begin{array}{l}\text { Primary } \\
\text { breast cancer }\end{array}$} & \multirow[t]{2}{*}{ Tertiles } & \multicolumn{3}{|c|}{ Apo A-1 } & \multicolumn{3}{|l|}{ Apo B } \\
\hline & & Events & Person-years & Unadjusted HR (95\% CI) & Events & Person-years & Unadjusted HR (95\% CI) \\
\hline \multicolumn{8}{|c|}{ Screening detected } \\
\hline & $\mathrm{T} 1$ & 11 & 677.99 & $(\operatorname{Ref})$ & 9 & 673.98 & (Ref) \\
\hline & $\mathrm{T} 2$ & 10 & 584.03 & $1.06(0.45-2.49)$ & 10 & 653.91 & $1.14(0.47-2.82)$ \\
\hline & $\mathrm{T} 3$ & 12 & 672.58 & $1.10(0.49-2.50)$ & 14 & 606.71 & $1.73(0.75-4.01)$ \\
\hline \multicolumn{8}{|c|}{ Clinically detected } \\
\hline & $\mathrm{T} 1$ & 13 & 552.16 & $(\operatorname{Ref})$ & 6 & 587.48 & (Ref) \\
\hline & $\mathrm{T} 2$ & 16 & 599.01 & $1.14(0.55-2.36)$ & 20 & 546.71 & $3.57(1.43-8.90)$ \\
\hline & $\mathrm{T} 3$ & 19 & 480.81 & $1.67(0.82-3.38)$ & 22 & 497.79 & $4.31(1.75-10.64)$ \\
\hline \multirow{2}{*}{$\begin{array}{l}\text { Primary } \\
\text { breast cancer }\end{array}$} & \multirow[t]{2}{*}{ Tertiles } & \multicolumn{3}{|c|}{ Apo A-1 } & \multicolumn{3}{|l|}{ Apo B } \\
\hline & & Events & Person-years & Adjusted HR (95\% CI)* & Events & Person-years & Adjusted HR $(95 \% \text { CI })^{\mathrm{a}}$ \\
\hline \multicolumn{8}{|c|}{ Screening detected } \\
\hline & $\mathrm{T} 1$ & 11 & 677.99 & & 9 & 673.98 & \\
\hline & $\mathrm{T} 2$ & 10 & 584.03 & $0.51(0.16-1.68)$ & 10 & 653.91 & $0.54(0.15-1.90)$ \\
\hline & $\mathrm{T} 3$ & 12 & 672.58 & $1.06(0.36-3.15)$ & 14 & 606.71 & $1.01(0.31-3.28)$ \\
\hline \multicolumn{8}{|c|}{ Clinically detected } \\
\hline & $\mathrm{T} 1$ & 13 & 552.16 & & 6 & 587.48 & \\
\hline & $\mathrm{T} 2$ & 16 & 599.01 & $1.98(0.84-4.71)$ & 20 & 546.71 & $3.40(1.19-9.72)$ \\
\hline & $\mathrm{T} 3$ & 19 & 480.81 & $1.40(0.59-3.34)$ & 22 & 497.79 & $3.87(1.39-10.75)$ \\
\hline
\end{tabular}

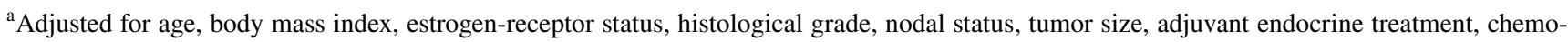
therapy, radiotherapy, surgery and statins

study, patients with high levels of Apo B are diagnosed with higher histological grade and larger tumors, which refutes the aforementioned hypothesis.

Measuring Apo B in blood is a part of routine cardiovascular care. It can detect if a patient is at risk of developing cardiovascular disease. We show here an association between Apo B and breast cancer outcome, and the second most common cause of death in breast cancer patients is cardiovascular disease [50]. Thus, it is needed to further investigate the role of apolipoproteins in breast cancer progression in order to answer the question if it is clinically relevant to assay for Apo B levels to predict risk of both BCR and cardiovascular disease in breast cancer patients. Future studies should longitudinally measure blood apolipoprotein levels after breast cancer diagnosis to detect possible changes in lipid metabolism by breast 
cancer while accounting for tumor, treatment, and patient characteristics.

\section{Conclusion}

High pre-diagnostic levels of Apo B were associated with an increased risk of recurrence among breast cancer patients. Circulating Apo A-1 was not associated with breast cancer outcome. The results warrant further study into the importance of lipid regulation in breast cancer patients.

Acknowledgements We acknowledge the support from Lund University Infrastructure grant "Malmö Population-based cohorts" (STYR 2019/2046). We acknowledge the assistance from Statistics Sweden (Statistiska Centralbyrån, SCB) for retrieving the death and/or emigration dates.

Author contributions Study concepts (SH, SB), study design ( $\mathrm{SH}$, TPA, SB), statistical analyses ( $\mathrm{SH})$, analyses of blood samples (OM), interpretation of data (SH, TPA, SB), manuscript preparation ( $\mathrm{SH}$, $\mathrm{SB}$ ), manuscript editing (SH), and manuscript review (TPA, DCF, MF, AHR, OM, SB). All authors read and approved the final manuscript.

Funding This work was supported by Aarhus University.

Availability of data and materials The data that support the findings of this study are available to appropriate academic parties upon reasonable request to the Malmö Preventative Project/ Malmö Diet and Cancer Study/Malmö Offspring Study steering committee (please see, https:// www.malmo-kohorter.lu.se/malmo-cohorts).

Code availability The code developed during this study is available upon reasonable request. Analyses were performed using Stata ${ }$, version 16 (StataCorp, College Station, Texas, USA).

\section{Declarations}

Conflict of interest All authors declare no conflicts of interest.

Ethical approval Ethical permission was obtained from the Ethical Committee at Lund University (D652/2005 and complement 501/2006 and 23/2007). Informed consent was obtained from all participants. The study was performed in accordance with relevant guidelines and regulations.

\section{Consent for publication Not applicable.}

Open Access This article is licensed under a Creative Commons Attribution 4.0 International License, which permits use, sharing, adaptation, distribution and reproduction in any medium or format, as long as you give appropriate credit to the original author(s) and the source, provide a link to the Creative Commons licence, and indicate if changes were made. The images or other third party material in this article are included in the article's Creative Commons licence, unless indicated otherwise in a credit line to the material. If material is not included in the article's Creative Commons licence and your intended use is not permitted by statutory regulation or exceeds the permitted use, you will need to obtain permission directly from the copyright holder. To view a copy of this licence, visit http://creativecommons.org/licenses/by/4.0/.

\section{References}

1. Bray F, Ferlay J, Soerjomataram I, Siegel RL, Torre LA, Jemal A (2018) Global cancer statistics 2018: GLOBOCAN estimates of incidence and mortality worldwide for 36 cancers in 185 countries. CA Cancer J Clin 68(6):394-424

2. DeSantis CE, Ma J, Sauer AG, Newman LA, Jemal A (2017) Breast cancer statistics, 2017, racial disparity in mortality by state. CA Cancer J Clin 67(6):439-448

3. Saphner T, Tormey DC, Gray R (1996) Annual hazard rates of recurrence for breast cancer after primary therapy. J Clin Oncol Off J Am Soc Clin Oncol 14(10):2738-2746

4. Lafourcade A, His M, Baglietto L, Boutron-Ruault M-C, Dossus L, Rondeau V (2018) Factors associated with breast cancer recurrences or mortality and dynamic prediction of death using history of cancer recurrences: the French E3N cohort. BMC Cancer 18(1): 171

5. Chan DSM, Vieira AR, Aune D, Bandera EV, Greenwood DC, McTiernan A et al (2014) Body mass index and survival in women with breast cancer-systematic literature review and meta-analysis of 82 follow-up studies. Ann Oncol Off J Eur Soc Med Oncol 25(10):1901-1914

6. Ren L, Yi J, Li W, Zheng X, Liu J, Wang J et al (2019) Apolipoproteins and cancer. Cancer Med 8(16):7032-7043

7. Emaus A, Veierød MB, Tretli S, Finstad SE, Selmer R, Furberg A-S et al (2010) Metabolic profile, physical activity, and mortality in breast cancer patients. Breast Cancer Res Treat 121(3):651-660

8. Buono G, Crispo A, Giuliano M, De Angelis C, Schettini F, Forestieri V et al (2020) Metabolic syndrome and early stage breast cancer outcome: results from a prospective observational study. Breast Cancer Res Treat 182(2):401-409

9. Oncoscience I The metastasis inducer CCN1 (CYR61) activates the fatty acid synthase (FASN)-driven lipogenic phenotype in breast cancer cells [Internet]. https://www.oncoscience.us/article/ 314/text/www.oncoscience.us/article/314/text/. Accessed 3 Aug 2021

10. Schug ZT, Peck B, Jones DT, Zhang Q, Grosskurth S, Alam IS et al (2015) Acetyl-CoA synthetase 2 promotes acetate utilization and maintains cancer cell growth under metabolic stress. Cancer Cell 27(1):57-71

11. Hilvo M, Orešiè AM (2012) Regulation of lipid metabolism in breast cancer provides diagnostic and therapeutic opportunities. Clin Lipidol 7(2):177-188

12. Huff T, Boyd B, Jialal I (2020) Physiology, Cholesterol. I: StatPearls [Internet]. Treasure Island (FL): StatPearls Publishing. http://www.ncbi.nlm.nih.gov/books/NBK470561/. Accessed 10 Feb 2021

13. Nelson ER, Chang C, McDonnell DP (2014) Cholesterol and breast cancer pathophysiology. Trends Endocrinol Metab 25(12):649-655

14. Feingold KR, Grunfeld C (2000) Introduction to lipids and lipoproteins. In: Feingold KR, Anawalt B, Boyce A, Chrousos G, de Herder WW, Dungan K et al. (eds) Endotext [Internet]. MDText. com, Inc., South Dartmouth (MA). http://www.ncbi.nlm.nih.gov/ books/NBK305896/. Accessed 1 Feb 2021

15. Rodrigues dos Santos C, Fonseca I, Dias S, Mendes de Almeida J (2014) Plasma level of LDL-cholesterol at diagnosis is a predictor factor of breast tumor progression. BMC Cancer 14(1):132

16. Li X, Tang H, Wang J, Xie X, Liu P, Kong Y et al (2017) The effect of preoperative serum triglycerides and high-density 
lipoprotein-cholesterol levels on the prognosis of breast cancer. The Breast 32:1-6

17. Lofterød T, Mortensen ES, Nalwoga H, Wilsgaard T, Frydenberg $\mathrm{H}$, Risberg $\mathrm{T}$ et al (2018) Impact of pre-diagnostic triglycerides and HDL-cholesterol on breast cancer recurrence and survival by breast cancer subtypes. BMC Cancer 18(1):654

18. Jung SM, Kang D, Guallar E, Yu J, Lee JE, Kim SW (2020) Impact of serum lipid on breast cancer recurrence. J Clin Med 9(9): 2846

19. Lee-Rueckert M, Escola-Gil JC, Kovanen PT (2016) HDL functionality in reverse cholesterol transport-Challenges in translating data emerging from mouse models to human disease. Biochim Biophys Acta 1861(7):566-583

20. Georgila K, Vyrla D, Drakos E (2019) Apolipoprotein A-I (ApoAI), immunity, inflammation and cancer. Cancers 11(8):1097

21. Cedó L, García-León A, Baila-Rueda L, Santos D, Grijalva V, Martínez-Cignoni MR et al (2016) ApoA-I mimetic administration, but not increased apoA-I-containing HDL, inhibits tumour growth in a mouse model of inherited breast cancer. Sci Rep 6(1):36387

22. Kimbung S, Chang C, Bendahl P-O, Dubois L, Thompson JW, McDonnell DP et al (2017) Impact of 27-hydroxylase (CYP27A1) and 27-hydroxycholesterol in breast cancer. Endocr Relat Cancer 24(7):339-349

23. Chang S-J, Hou M-F, Tsai S-M, Wu S-H, Hou LA, Ma H et al (2007) The association between lipid profiles and breast cancer among Taiwanese women. Clin Chem Lab Med 45(9):1219-1223

24. His M, Zelek L, Deschasaux M, Pouchieu C, Kesse-Guyot E, Hercberg S et al (2014) Prospective associations between serum biomarkers of lipid metabolism and overall, breast and prostate cancer risk. Eur J Epidemiol 29(2):119-132

25. Borgquist S, Butt T, Almgren P, Shiffman D, Stocks T, Orho-Melander $\mathrm{M}$ et al (2016) Apolipoproteins, lipids and risk of cancer. Int J Cancer 138(11):2648-2656

26. Huang H-L, Stasyk T, Morandell S, Dieplinger H, Falkensammer $\mathrm{G}$, Griesmacher A et al (2006) Biomarker discovery in breast cancer serum using 2-D differential gel electrophoresis/MALDI-TOF/ TOF and data validation by routine clinical assays. Electrophoresis 27(8):1641-1650

27. Devaraj S, Semaan JR, Jialal I (2020) Biochemistry, apolipoprotein B. I: StatPearls [Internet]. StatPearls Publishing, Treasure Island (FL). http://www.ncbi.nlm.nih.gov/books/NBK538139/. 1 Feb 2021

28. Contois JH, McConnell JP, Sethi AA, Csako G, Devaraj S, Hoefner DM et al (2009) Apolipoprotein B and cardiovascular disease risk: position statement from the AACC Lipoproteins and Vascular Diseases Division Working Group on Best Practices. Clin Chem. Marts 55(3):407-419

29. Lee G, Jeong YS, Kim DW, Kwak MJ, Koh J, Joo EW et al (2018) Clinical significance of APOB inactivation in hepatocellular carcinoma. Exp Mol Med 50(11):1-12

30. Apolipoprotein B but not LDL cholesterol is associated with coronary artery calcification in type 2 diabetic whites I diabetes [Internet]. https://diabetes.diabetesjournals.org/content/58/8/1887. Accessed 1 Feb 2021

31. Sniderman AD, Thanassoulis G, Glavinovic T, Navar AM, Pencina M, Catapano A et al (2019) Apolipoprotein B particles and cardiovascular disease: a narrative review. JAMA Cardiol 4(12): 1287

32. Lim Y, Yoo S, Lee SA, Chin SO, Heo D, Moon JC et al (2015) Apolipoprotein B is related to metabolic syndrome independently of low density lipoprotein cholesterol in patients with type 2 diabetes. Endocrinol Metab 30(2):208-215

33. Koene RJ, Prizment AE, Blaes A, Konety SH (2016) Shared risk factors in cardiovascular disease and cancer. Circulation 133(11):1104-1114
34. Chandler PD, Song Y, Lin J, Zhang S, Sesso HD, Mora S et al (2016) Lipid biomarkers and long-term risk of cancer in the Women's Health Study. Am J Clin Nutr 103(6):1397-1407

35. Berglund G, Elmståhl S, Janzon L, Larsson SA (1993) Design and feasibility. J Intern Med 233(1):45-51

36. Manjer J, Carlsson S, Elmståhl S, Gullberg B, Janzon L, Lindström M et al (2001) The Malmö Diet and Cancer Study: representativity, cancer incidence and mortality in participants and non-participants. Eur J Cancer Prev Off J Eur Cancer Prev Organ ECP 10(6):489-499

37. Wettermark B, Hammar N, Fored CM, MichaelFored C, Leimanis A, Otterblad Olausson P et al (2007) The new Swedish Prescribed Drug Register-opportunities for pharmacoepidemiological research and experience from the first six months. Pharmacoepidemiol Drug Saf 16(7):726-735

38. Brooke HL, Talbäck M, Hörnblad J, Johansson LA, Ludvigsson JF, Druid H et al (2017) The Swedish cause of death register. Eur J Epidemiol 32(9):765-773

39. Ludvigsson JF, Almqvist C, Bonamy A-KE, Ljung R, Michaëlsson K, Neovius M et al (2016) Registers of the Swedish total population and their use in medical research. Eur J Epidemiol 31(2): 125-136

40. Pero RW, Olsson A, Berglund G, Janzon L, Larsson SA, Elmståhl S (1993) The Malmö biological bank. J Intern Med 233(1):63-67

41. Obesity and overweight [Internet]. https://www.who.int/newsroom/fact-sheets/detail/obesity-and-overweight. 2021. https:// www.who.int/news-room/fact-sheets/detail/obesity-and-overw eight. Accessed 16 Nov 2020

42. Cong L, Liu Q, Zhang R, Cui M, Zhang X, Gao X et al (2018) Tumor size classification of the 8th edition of TNM staging system is superior to that of the 7 th edition in predicting the survival outcome of pancreatic cancer patients after radical resection and adjuvant chemotherapy. Sci Rep 8:10383

43. Bloom HJG, Richardson WW (1957) Histological grading and prognosis in breast cancer. Br J Cancer 11(3):359-377

44. Pan H, Gray R, Braybrooke J, Davies C, Taylor C, McGale P et al (2017) 20-Year risks of breast-cancer recurrence after stopping endocrine therapy at 5 years. N Engl J Med 377(19):1836-1846

45. Morales M, Santana N, Soria A, Mosquera A, Ordovás J, Nóvoa J et al (1996) Effects of tamoxifen on serum lipid and apolipoprotein levels in postmenopausal patients with breast cancer. Breast Cancer Res Treat 40(3):265-270

46. Elisaf MS, Bairaktari ET, Nicolaides C, Kakaidi B, Tzallas CS, Katsaraki A et al (2001) Effect of letrozole on the lipid profile in postmenopausal women with breast cancer. Eur J Cancer 37(12):1510-1513

47. McCormack VA, Silva IS (2006) Breast density and parenchymal patterns as markers of breast cancer risk: a meta-analysis. Cancer Epidemiol Prev Biomark 15(6):1159-1169

48. Ding J, Warren R, Warsi I, Day N, Thompson D, Brady M et al (2008) Evaluating the effectiveness of using standard mammogram form to predict breast cancer risk: case-control study. Cancer Epidemiol Prev Biomark 17(5):1074-1081

49. Boyd NF, Connelly P, Byng J, Yaffe M, Draper H, Little L et al (1995) Plasma lipids, lipoproteins, and mammographic densities. Cancer Epidemiol Prev Biomark 4(7):727-733

50. Afifi AM, Saad AM, Al-Husseini MJ, Elmehrath AO, Northfelt DW, Sonbol MB (2020) Causes of death after breast cancer diagnosis: a US population-based analysis. Cancer 126(7):1559-1567

Publisher's Note Springer Nature remains neutral with regard to jurisdictional claims in published maps and institutional affiliations. 